\title{
CPU Performance in Data Migrating from Virtual Machine to Physical Machine in Cloud Computing
}

\author{
Lochan.B \\ CMR University \\ Bangalore, India
}

\author{
Dr. Divyashree B A \\ Dept of CSE,BNMIT \\ Bangalore, India
}

\begin{abstract}
Cloud computing has a massive use of virtual machines to permit isolated workload to be used from one resource to the another and resource usages to be controlled. Migrating from one operating system to the other operating system is difficult. The virtual machines mainly deals with the live migration process. In this paper, we present the Performance of CPU in Virtual Machine with various features like Cluster, CPU, Live migration, Data Centers, Hosts, Storage, Disks, Templates. The multiprocessor is mainly used in the host machine which allow the features of guest operating system. There are various performance anomalies, which overheads for the infrastructure for the cloud. They are various implication for the results in the future architecture for the cloud infrastructure. Both the container and virtual machine support for the input output intensive application from future cloud allocated to the different application. The large number of the storage and network activity has to served for challenges on the platform. Cloud Computing in the virtual machine has high consumption of memory and CPU resource for inefficient virtualization software.
\end{abstract}

Keywords: Live Migration, Cluster, Virtualization, Host, Data Centers

\section{INTRODUCTION}

Virtual Memory are used massively in the cloud computing. It performs the platform like Hypervisor (Hyper-V) that the virtual machines are available for the customers to run the service like Platform as a Service(Paas) that make the available for customer to run inside the workloads running inside the virtual memory[1]. It has a various challenge for preventing and diagnose the performance anomalies in virtualized environment. The Application running inside black box will have infrastructure as the environment. Cloud Management has to work automatically that prevents the performance anomaly for the management for Virtualized Cloud System. There are two main objective to achieve the system level metrics i) A performance anomaly that can have raise detection alerts. ii) The system metrics that cause inference grained faculty virtual machine to the performance anomaly [2]. Virtual Machine in the multiprocessor allows the guest operating system for the use of the symmetric multiprocessor that are used in the host machine. In these, it mainly consists of virtual machine monitor and performance of the overhead of the virtualization. It has various types of workloads for the physical host resources for the encapsulated in a virtual machine run on a single physical host. They are various Virtualization and Symmetric multiprogramming technologies are having opposite goals. Some of the example for Software Virtualization are Microsoft Hyper-V, Xen, VirtualBox, VMware ESX/ESXi. In Virtualization multiple operating system are done in concurrently as shown in Fig.1.

\begin{tabular}{|c|c|c|c|}
\hline \multicolumn{2}{|c|}{ Application } & \multicolumn{2}{|c|}{ Application } \\
\hline \multicolumn{2}{|c|}{ Operating Systems } & \multicolumn{2}{|c|}{ Operating System } \\
\hline \multicolumn{2}{|c|}{ Virtualized Hardware } & \multicolumn{2}{|c|}{ Virtualized Hardware } \\
\hline \multicolumn{4}{|c|}{ Hypervisor (Virtualization) } \\
\hline CPU & MEM & NIC & DISK \\
\hline
\end{tabular}

Fig. 1. Virtualization

Several techniques are mainly used for the reduce of virtual machine monitor intervention. The multiple operation system has to share a single utilization of system resources like Input/Output device. There are many approaches are used for the enabling virtualization. Full Virtualization and Para Virtualization. Full Virtualization is mainly used for modifications that are not used in the hardware support such as technology used for the virtualization. Para Virtualization has operating system to work with modification of virtual machine monitor. Input or output needs some effectively handling virtualization to be improved for the scalability such as guest access for the $\mathrm{I} / \mathrm{O}$ devices. The virtual machine used to overhead the saturate the high throughput situation such as virtual machine. Single Root Virtualization has Direct I/O virtualization for the major device resource to achieve both sharing and high performance[3].

\section{BACKGROUND}

\subsection{Requirement and Motivation for Cloud Virtualization:}

Every user and program should operate using the privileges to complete the job. The least Common mechanism has strongly implement the principles of least privileges. Shared library can be especially have modern application require different version of the library. The requirements are mainly used to have a resource isolation for suitable infrastructure or configuration between application. It improves the I/O concurrency for enhanced storage 
performance. It has hardware support for unit virtualization for memory

\subsection{Scientific Computing:}

The Scientific Computing has heterogeneous CPU, Memory and Network as the bottleneck resource. The average of the Scientific Cluster is found to be around many nodes to be stable over the past many years. The Network, Memory, I/O, CPU can have Heterogeneous as the bottleneck resources. The Workloads are in scientific and users in performance of cloud computing service. It mainly depends on the performance in which the focus of the work to be carried out[4]. The Characteristics Performance of four commercial cloud computing service has been executing parallel application of up to many processor.

\subsection{Live Migration}

It is the process of moving a guest virtual machine from one host physical machine to another in Fig.2. It is mainly used for load balancing it can be moved from host physical machine with lower usage the physical machine it has overload to add, upgrade or remove hardware device in the host physical machine it can have an hardware independence in the hardware improvements. Energy Saving helps for guest virtual machine should be powered off to save energy and low cost in usage of periods. Geographic Migration is the guest virtual machine that can be moved from one location to the lower latency or in serious circumstances. The main features is to work for sending the state of the guest virtualized device or the machine memory it has to be migrated. It is also recommended to use live migration from migration from virtual machine[5].

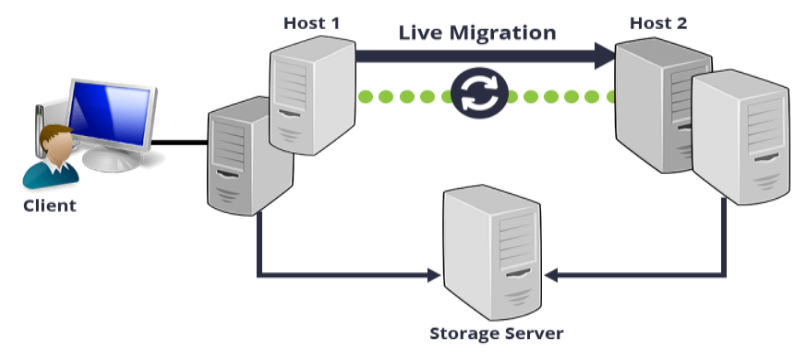

Fig2: Live migration from one host to the another host

\section{SYSTEM DESIGN}

Hypervisor has its own policy for what a virtual machine have its CPU by default. It has to decide the some hypervisor which CPU host Physical machine for the available for the guest virtual machine. The host has its own filtering, Classifying the Physical CPU model for each group has its own virtual machine[6]. It has a safe migration between the host physical machine that provided the physical CPU that classify the same group for a suitable guest virtual machine model is successful between host physical machine. It has to emulate features that is aware of the features that were created after the hypervisor was released may not. It has the grouping and allocation of resources on per guest virtual machine[7].

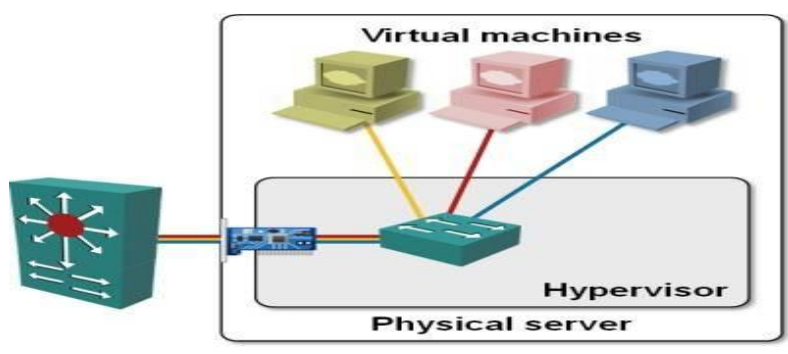

Fig.3 : Live migration networking

The System Administrator to in the either turntables against the guest operating system. It mainly consists of i) Memory: The memory controller allows for setting limits on RAM and swap usage and querying cumulative usage of all processes in the group. ii) CPU: The CPU scheduler controller controls the prioritization of processes in the group. It is similar to grant level privileges. iii) Freezer: The freezer controller pauses and resumes execution of processes in the group[8]. This is similar to SIGSTOP for the whole group. iii) Devices : The devices controller grants access control lists on character and block devices in fig. 3

\section{EVALUATION}

The numerous aspects can be done in the performance issue on the non-virtualized execution because it has various resources available for the productive work. When one or more resource are fully utilized and have a workload metrics like throughput and the latency for the overhead virtualization[9]. All the test are performed on the system with 8GB RAM. The two system are needed for calculating the performance of live migration from one operating system to the other operating system[10]. In Virtualization technology, while deploying it must ensure that Operating System and physical machine cannot be compromised. The guest Virtual Machine, Network, Memory, Devices system have security on system using virtualization using the deployment plan. There are various works are carried for the state the guest virtual machine's memory and device for a destination from host physical machine. The main features is to use shared, networked storage for the guest virtual machine to be migrated. In these shared storage, while migration can be performed live or not. In a live migration, the guest virtual machine continue to run in the host physical machine for which it can have memory pages from the host physical machine its destination host physical machine[11]. During migration the source for any changes it can be already transferred for the initial pages to be transferred. A migration is not performed live, it has to suspends for the already transferred and to transfer the initial pages to been transferred. If the network is experiencing the use or low bandwidth, the migration will take more time. The destination host physical machine it has the offline migration to be used as the live migration will be complete such as migration depends on the network bandwidth and latency[12]. 


\begin{tabular}{|l|l|}
\hline System & PowerEdge servers \\
\hline CPU & $2 *$ Quad Core Intel \\
\hline Memory & 8 GB RAM \\
\hline RAM Per VM & 1.5 GB \\
\hline Operating System & Hyper-V \\
\hline Disk Drive & $4 * 146$ GB 10,000 RPM \\
\hline
\end{tabular}

Table 1: System Configuration

\subsection{HARDWARE}

Table 1. shows the system configuration for client hardware as Single-socket, quad core server,2.50GHZ Intel E420 Processor. 8GB Memory. All Experiments are conducted in the number of System for a CPU Virtual Machine having the number of pCPUs used equals to the number of vCPUs configured in the virtual machine[13]. Fig.4 shows RHEV-H Networking configuration. Most of the virtualization platforms has interface that allows the guest virtual machine for the hypervisor as guest virtual machine system level metric from the usage from domain0[14].

\subsection{SOFTWARE}

The software is mainly used as Virtual Box. In these, Hypervisor is mainly used. The scripting language is used i.e python.

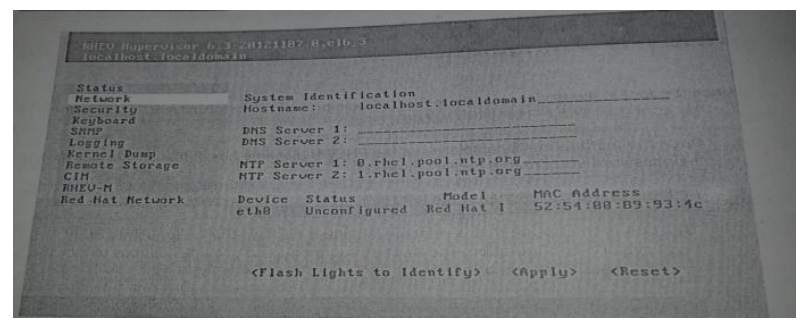

\section{Fig4. RHEV-H Networking Configuration}

\section{CONCLUSION AND FUTURE WORK}

Virtualization is important technology because it has more efficient use of resource. It differs from a physical system that leads to degradation for application that is running on a virtual Machine. The Performance of the data migration from one operating system to the other operating system to carried out is difficult. The virtual machine has the performance of input output to negligible when it is has a large rates. It contains the tradeoff started with the overhead of high packet rates. It represent a tradeoff between of management and performance should be case-by-case basis. It has the limited of each workload to single socket performance of turning and analysis. This leads to several isolation of multiple workload on the same server, live resizing of containers and virtual machine of scale out and tradeoffs between restarting and live migration. The innovation of the Virtualization has to bring the performance of the more data in the Para Virtualization in the future.

\section{REFERENCES}

[1] Wes Felter, Alexandre Ferreira, Ram Rajamony, Juan Rubio by IBM Research, Austin, Tx by “ An updated Performance Comparison of Virtual machine and Linux Containers" Vol.4 2014

[2] Yongmin Tan, Hiep Nguyen, Zhiming Shen, Xiahui Gu North Crohina State Univerity "Prepare:Predictive Performance Anomaly Prevention for Virtualized Cloud System" page 241-254 2013

[3] Yaozu Dong, Xiaowei Yang,Xiaoyong by intel china software center "High Performance Network Virtualization with SR-IOV “

[4] Alexandru Losup "Performance Analysis of Cloud Computing Services for many tasks scientific computing"

[5] http://www.vmware.com/pdf/virtualization.pdf "Virtualization Overview"

[6] Advanced multi layered unification file system. http://aufs.sourceforgenet,2014

[7] “Virtual computing lab,” http://vcl.ncsu.edu/.

[8] Ari Balogh, Google Compute Engine is now generally available with expanded OS support, transparent maintenance and lower price. http://googledevelopers.blogsport.com /2013/12/ google-compute-engine-is-now-generally.html Dec2013

[9]Jerome H.Saltzer and Micheal D.Schroeder The protection of information in computer system. In proceeding of the IEEE,Vol 63 Sep 2013

[10] “Amazon Elastic Compute Cloud," http://aws. amazon. com/ec2/

[11] "Rubis Online Auction System,"http://rubis.ow2.org/.

[12] C.Wang, V.Talwar, K.Schwan and P.Ranganathan, "online detection of utility cloud anomalies using metric distribution," in proc. of NOMS,2010

[13] D.Chisnall, "The definition guide to the XEN"

[14] G.J.Popek and R.P.Goldberg, Formal requirements for virtualizable third generation architecture communications of the ACM-July 1974 